as they must be personalized and can be extremely toxic. Furthermore, there has been limited success in treating solid cancers with them.

Klichinsky et al. were able to engineer macrophages expressing a CAR and show that in humanized mouse models, infusion with this resulted in a proinflammatory tumor microenvironment that activated $T$ cells. In a clinical trial using CAR natural killer cells, 11 patients with CD19+ cancer were treated, and the large majority responded to the treatment without toxic effects. A new era for CARs in cancer therapy has commenced.

https://doi.org/10.1038/s41591-020-01151-2

\section{REGENERATIVE MEDICINE}

\section{A personal approach for Parkinson's disease}

N. Engl. J. Med. 382, 1926-1932 (2020)

A tailored-therapy approach with induced pluripotent stem cells improved the symptoms of a patient's Parkinson's disease.

Parkinson's disease is a progressive, debilitating disease and is linked to neuronal loss in the substantia nigra, which causes striatal dopamine deficiency. Approaches attempting to replace or regenerate these neurons have been studied for a long time; however, nothing has proven effective.

A group of researchers in the USA were able to derive induced pluripotent stem cells from a patient with Parkinson's disease and were able to differentiate these into dopaminergic progenitor cells. Two separate transfers of these cells into the patient's brain were made with no immunosuppressants. At 18-24 months after the transfers, there was an improvement in the patient's symptoms without any adverse effects. The approach needs to be tested further in a clinical trial and needs to be analyzed after additional follow-up before it can be considered for clinical use.

https://doi.org/10.1038/s41591-020-01152-1

\section{VACCINES \\ WHO declares Africa polio-free}

World Health Organization https://www.who.int/ news/item/25-08-2020-global-polio-eradicationinitiative-applauds-who-african-region-for-wildpolio-free-certification (2020)

Lancet Glob. Health 8, e1345-e1351 (2020)

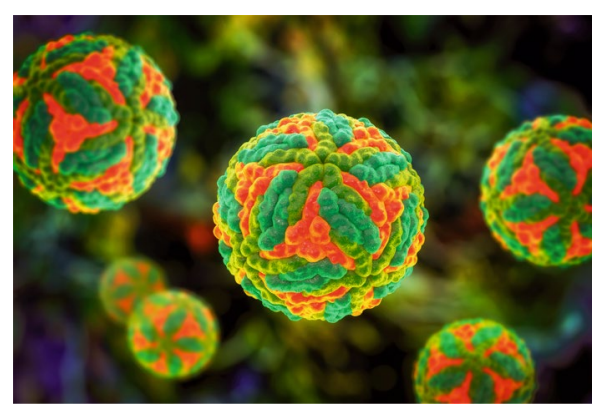

Credit: KATERYNA KON/SCIENCE PHOTO LIBRARY / Getty

On 25 August 2020, the Africa Regional Commission for the Certification of Poliomyelitis Eradication declared the African region polio-free.

Polio is a serious disease and can lead to lifetime of paralysis or even death. A global initiative was started in 1988 with the aim of eradicating the spread of wild poliovirus.

To be certified polio-free, a region has to have gone 3 years without the virus being isolated from a person with paralysis, and in Africa this was from a person in Nigeria in 2016. This leaves just two countries worldwide in which there is still transmission of wild poliovirus.

HS

https://doi.org/10.1038/s41591-020-01156-x

An insulin analog can be taken once per week to regulate glucose levels in people with type 2 diabetes.

Those who develop type 2 diabetes are often eventually prescribed insulin to regulate glucose levels. However, the once-daily need for this treatment is off-putting, and it is thought that acceptance of and adherence to insulin treatment might be increased if the frequency of administration could be reduced.

Julio Rosenstock and colleagues carried out a phase 2 trial of icodec, an insulin analog that is taken once per week. In the trial of 247 participants, those assigned icodec showed no more adverse events than did those taking an alternative daily insulin, and iodec was able to lower glucose. This quality-of-life alteration could be transformative for those with type 2 diabetes.

https://doi.org/10.1038/s41591-020-01153-0

\section{SOCIAL JUSTICE}

HS Science strikes for Black lives

https://www.particlesforjustice.org/

The killing of George Floyd, a Black man, by a white police officer in Minneapolis, Minnesota, on 25 May 2020, and the deaths of countless other Black people at the hands of the police, combined with the disproportionate death toll of COVID-19 on Black and minority ethnic people, called the scientific and medical communities to self-reflect on pervasive systemic racism in their communities.

On 10 June, many in the scientific community joined a strike for Black lives, to take time to undergo overdue anti-racist self-education and to give Black academics who have carried this burden a day off. Elsewhere on Twitter, \#blackinstem and other hashtags associated with scientific areas of research have arisen, raising the profiles of Black researchers, and highlighting the racist barriers they must overcome.

https://doi.org/10.1038/s41591-020-01155-y

\section{GENE THERAPY \\ RNA therapies prepare for the spotlight}

N. Engl. J. Med. 382, 2289-2301 (2020)

N. Engl. J. Med. 383, 151-158 (2020)

N. Engl. J. Med. 383, 109-119 (2020)

2020 saw several RNA-based therapies show promise, including in clinical trials.

Acute hepatic porphyria is a set of rare diseases in which porphyrins build up, which results in neurotoxic effects. Intermittent hepatic porphyria is the most common form and is caused by upregulation of hepatic $\delta$-aminolevulinic acid synthase 1 . A phase 3 trial of givosiran, an RNA-interference therapy that targets the gene encoding this enzyme, resulted in a reduced rate of porphyria attacks.

A mutation in SOD1 (which encodes superoxide dismutase) causes $10 \%$ of cases of amyotrophic lateral sclerosis. A phase $1 / 2$ trial of intrathecal administration of an antisense oligonucleotide, tofersen, showed that this approach results in a reduction in SOD1 levels when administered. A separate study of two patients shows that an anti-SOD1 microRNA reduced the expression of SOD1 in two patients with amyotrophic lateral sclerosis. It finds that the approach is safe and has shown some clinical promise in one of the patients. HS https://doi.org/10.1038/s41591-020-01154-Z 